\title{
FGFR Family Alteration Negative
}

National Cancer Institute

\section{Source}

National Cancer Institute. FGFR Family Alteration Negative. NCI Thesaurus. Code C150613.

An indication that mutation, overexpression, fusion or rearrangement of an FGFR family member has not been detected in a sample. 